\title{
MÉTODO PARA DETERMINAÇÃO DE HEPTACLORO, ALDRIN, DIELDRIN E ENDRIN EM PRÓPOLIS *
}

\author{
MARIA LÚCIA RIBEIRO ** \\ OSMAR MALASPINA *** \\ ELAINE DE FÁTIMA GALATTI JARDIM **** \\ LUCIANA POLESE \\ MARIO SERGIO PALMA *** \\ SANDRO NAVICKIENE
}

\begin{abstract}
Desenvolveu-se método para determinação de Heptacloro, Aldrin, Dieldrin e Endrin em própolis (sólida e solução). Após homogeneização da amostra com Celite, a extração dos pesticidas foi efetuada com hexano e o extrato purificado em cartucho de Florisil. A eluição dos pesticidas foi realizada com hexano:diclorometano $(9: 1 \mathrm{v} / \mathrm{v})$ e o eluato concentrado e analisado por cromatografia em fase gasosa com detecção por captura de elétrons (CG-DCE). A eficiência do método proposto foi demonstrada pelos valores médios de recuperação entre 79 e 100\%, com valores de desvio padrão relativo entre 2,7 e $16,6 \%$, obtidos nas análises de recuperação realizadas com amostras fortificadas nos níveis 0,6 e $1,2 \mu \mathrm{g} / \mathrm{g}$.
\end{abstract}

PALAVRAS-CHAVE: ALDRIN; DIELDRIN; ENDRIN; HEPTACLORO; PRÓPOLIS.

\section{INTRODUÇÃO}

Própolis é um produto elaborado em colméias a partir de substâncias resinosas e balsâmicas que as abelhas coletam de brotos, exsudados

\footnotetext{
* Trabalho desenvolvido na Universidade Estadual Paulista (UNESP), Instituto de Química, Departamento de Química Orgânica, Araraquara, SP, Brasil.

** Professora Dra. do Departamento de Química Orgânica UNESP, Araraquara, SP. (e-mail: mlucia@iq.unesp.br).

*** Professores Doutores do Centro de Estudos de Insetos Sociais, Instituto de Biociências, UNESP, Rio Claro, SP. (http://www.rc.unesp.br/ib/ceis).

**** Servidor Técnico-administrativo, UNESP, Araraquara, SP.

***** Pós-graduando em Química, UNESP, Araraquara, SP.
} 
de árvores e de outras partes do tecido vegetal. As abelhas coletam esse material com o uso de suas mandíbulas e adicionam secreções salivares e ceras para a elaboração final da própolis. A cor, o sabor, o odor e a consistência da própolis dependem das espécies vegetais das quais provêem $(1,2)$. Análises laboratoriais (2) revelaram que a própolis consiste de resinas e bálsamos (55\%), cera (30\%), óleos voláteis (10\%) e pólen $(0,5 \%)$. Este produto apícola é muito utilizado devido às suas propriedades antiinflamatórias, antioxidantes, anestésicas, estimulantes, anti-sépticas e cicatrizantes (1). Também é eficiente no tratamento de desordens respiratórias causadas por bactérias e fungos que levam à deterioração das condições nutricionais e imunológicas de pacientes (3). Comercialmente, a própolis é vendida em solução alcoólica em concentrações variáveis (4).

Métodos para a determinação de resíduos de várias classes de pesticidas em matrizes de mel (5-9), cera de abelha (5) e pólen $(5,10)$, empregando cromatografia a gás (CG) e/ou cromatografia em fase líquida de alta eficiência (CLAE), têm sido desenvolvidos. Entretanto, nenhum procedimento experimental para a determinação de resíduos de pesticidas em própolis foi relatado na literatura consultada.

Neste trabalho descreve-se metodologia analítica rápida e eficiente para a determinação de resíduos de quatro pesticidas organoclorados (Heptacloro, Aldrin, Dieldrin e Endrin) em própolis (sólida e solução).

\section{MATERIAL E MÉTODOS}

\subsection{REAGENTES E SOLVENTES}

Foram utilizados hexano e diclorometano (nanograde, Mallinckrodt); cartuchos para extração em fase sólida Supelclean ${ }^{\mathrm{TM}}{ }^{\text {Florisil }}$ (500 mg/3 mL, Supelco) e Celite (Reagen). Os padrões de Heptacloro, Aldrin, Dieldrin e Endrin foram fornecidos pela U.S. Environmental Protection Agency (EPA), Research Triangle Park, NC, USA. Soluções estoque de cada pesticida $(0,2 \mu \mathrm{g} / \mu \mathrm{L})$ foram preparadas em hexano. Uma solução intermediária contendo todos os pesticidas $(0,01 \mu \mathrm{g} / \mu \mathrm{L})$ foi preparada por diluição das soluções estoque em hexano. A solução de fortificação contendo $0,001 \mu \mathrm{g} / \mu \mathrm{L}$ de cada pesticida foi preparada por diluição da solução intermediária em hexano. As soluções padrão, empregadas nas análises cromatográficas, foram preparadas por adição da solução de fortificação aos extratos das amostras não-fortificadas. Os padrões e as soluções dos pesticidas foram armazenados em freezer $\left(-18^{\circ} \mathrm{C}\right)$. 


\subsection{EQUIPAMENTO}

Cromatógrafo a gás (modelo 3300, Varian), equipado com detector por captura de elétrons ( $\left.{ }^{63} \mathrm{Ni}\right)$, injetor "on column" (Modelo 1040, Varian), coluna de sílica fundida Megabore ${ }^{\circledR}$ (15 m comprimento $\times$ 0,53 mm diâmetro interno $\times 0,83 \mu \mathrm{m}$ espessura do filme, J\&W Scientific), recoberta com a fase líquida DB-608 (5\% difenil 95\% dimetilpolissiloxano) e integrador (modelo 4290, Varian). Condições de operação: temperatura do injetor = $240^{\circ} \mathrm{C}$; temperatura do detector $=300^{\circ} \mathrm{C}$, programação de temperatura da coluna: inicial $140^{\circ} \mathrm{C}$ (2 min), primeira rampa de temperatura de $140^{\circ} \mathrm{C}$ a $240^{\circ} \mathrm{C}\left(10^{\circ} \mathrm{C} / \mathrm{min}\right)$, permanecendo por $5 \mathrm{~min}$. Segunda rampa de aquecimento de $240^{\circ} \mathrm{C}$ a $265^{\circ} \mathrm{C}\left(5^{\circ} \mathrm{C} / \mathrm{min}\right)$, permanecendo por $10 \mathrm{~min}$ em $265^{\circ} \mathrm{C}$; vazão do gás (Nitrogênio): $30 \mathrm{~mL} / \mathrm{min}$ (coluna + detector); vazão do gás de arraste (Nitrogênio) $=2 \mathrm{~mL} / \mathrm{min}$ (coluna) e volume injetado $=1 \mu \mathrm{L}$.

\subsection{PREPARAÇÃO E FORTIFICAÇÃO DAS AMOSTRAS}

Efetuou-se a homogeneização de 2,0 g de própolis (sólida ou solução alcoólica 30 \%) com 4,0 g de Celite.

A fortificação das amostras foi realizada por adição de $100 \mu \mathrm{L}$ (nível de fortificação: $0,6 \mu \mathrm{g} / \mathrm{g}$ ) e $200 \mu \mathrm{L}$ (nível de fortificação: $1,2 \mu \mathrm{g} / \mathrm{g}$ ) de solução padrão a $0,5 \mathrm{~g}$ da mistura de própolis e Celite. Após a fortificação, as amostras foram submetidas ao procedimento analítico.

\subsection{PROCEDIMENTO ANALÍTICO}

Submeteu-se $0,5 \mathrm{~g}$ da mistura de própolis e Celite à extração com $10 \mathrm{~mL}$ de hexano, durante 20 min em agitador mecânico (Maxi-Mix IIIM, Thermolyne). O procedimento de extração foi repetido com $10 \mathrm{~mL}$ de hexano, o extrato concentrado até, aproximadamente, $2 \mathrm{~mL}$ em evaporador rotatório $\left(40^{\circ} \mathrm{C}\right)$ e transferido para cartucho de Florisil (conectado a um Vacuum Manifold, Supelco), previamente condicionado com $5 \mathrm{~mL}$ de hexano. A eluição dos pesticidas foi processada com $10 \mathrm{~mL}$ de hexano:diclorometano $(9: 1 \mathrm{v} / \mathrm{v})$. O eluato foi concentrado até, aproximadamente, $1 \mathrm{~mL}\left(40^{\circ} \mathrm{C}\right)$ e o volume ajustado a $10,0 \mathrm{~mL}$ com hexano.

\subsection{ANÁLISES CROMATOGRÁFICAS}

As determinações qualitativas e quantitativas foram realizadas pela comparação dos dados cromatográficos obtidos com as soluções 
padrão, extratos das amostras fortificadas e não-fortificadas analisados sucessivamente por cromatografia a gás, com detecção por captura de elétrons (CG-DCE).

\subsection{PROCEDIMENTO ESTATÍSTICO}

Os valores de limite de detecção e de quantificação do método foram calculados segundo procedimento estatístico descrito por THIER e ZEUMER (11).

\section{RESULTADOS E DISCUSSÃO}

No presente estudo, a seleção dos quatro pesticidas organoclorados foi realizada com base nas solicitações das empresas brasileiras exportadoras de produtos apícolas. Embora o uso da maioria dos pesticidas organoclorados tenha sido proibido na década de oitenta é importante considerar sua persistência no ambiente e a possibilidade do comércio ilegal destes pesticidas.

Na primeira etapa, procedimentos "on-line" (12-14), cuja extração dos pesticidas e a purificação dos extratos são realizadas em uma única etapa, testaram-se colunas "home-glass", empacotadas com diferentes adsorventes (alumina, alumina/sílica, sílica). Resultados insatisfatórios demonstraram a necessidade de etapa preliminar de extração. Entre os solventes testados para a extração, o hexano forneceu extratos mais adequados para a purificação em cartuchos de Florisil. Quatro sistemas de solventes foram testados para a eluição dos pesticidas em cartuchos de Florisil, cujos resultados das análises de recuperação, realizadas com amostras de própolis (solução) fortificadas são apresentados na Tabela 1. Empregando $10 \mathrm{~mL}$ dos sistemas $\mathrm{A}$ (hexano) e $\mathrm{B}$ (hexano:diclorometano 9,5:0,5 v/v) valores de recuperação adequados (84 a 116\%) foram obtidos apenas para Heptacloro e Aldrin. Valores médios de recuperação satisfatórios (82 a 116\%) foram obtidos para Dieldrin e Endrin empregandose volume adicional $(10 \mathrm{~mL})$ dos sistemas de solventes mencionados. Os resultados de recuperação obtidos quando apenas $10 \mathrm{~mL}$ dos sistemas $\mathrm{C}$ (hexano:diclorometano 9:1 v/v) e D (hexano:diclorometano 8:2 v/v) foram empregados, variaram entre 71 e 109\%. Demonstraram assim a necessidade de alteração da polaridade do solvente, visando promover a redução do volume de solvente e a eluição quantitativa de Dieldrin e Endrin. Tendo em vista que os cromatogramas dos extratos obtidos com o sistema D apresentaram muitos compostos interferentes, o sistema C hexano:diclorometano 9:1 v/v foi selecionado para a eluição dos pesticidas. 


\section{TABELA 1 - EFICIÊNCIA DA RECUPERAÇÃO EMPREGANDO-SE DIFERENTES SISTEMAS DE SOLVENTES PARA A ELUIÇÃO DOS PESTICIDAS}

\begin{tabular}{|c|c|c|c|c|c|}
\hline \multirow[t]{2}{*}{ Pesticida } & \multirow[t]{2}{*}{ 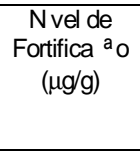 } & \multicolumn{3}{|c|}{$\begin{array}{c}\text { Recupera }{ }^{a} \text { o } \\
\text { MØdia } \\
\text { (Intervalo) }\end{array}$} & \multirow[b]{2}{*}{ Sistema $\mathrm{D}^{\mathrm{d}}$} \\
\hline & & Sistema $A^{a}$ & Sistema B $^{\mathrm{b}}$ & Sistema C $^{\mathrm{C}}$ & \\
\hline Heptacloro & 1,2 & $\begin{array}{c}109^{\mathrm{e}} \\
(105-113)\end{array}$ & $\begin{array}{c}89^{e} \\
(84-94)\end{array}$ & $\begin{array}{c}84 \\
(75-93)\end{array}$ & $\begin{array}{c}83 \\
(75-90)\end{array}$ \\
\hline Aldrin & 1,2 & $\begin{array}{c}111^{\mathrm{e}} \\
(104-116)\end{array}$ & $\begin{array}{c}99^{\mathrm{e}} \\
(95-102)\end{array}$ & $\begin{array}{c}71 \\
(70-72)\end{array}$ & $\begin{array}{c}109 \\
(99-117)\end{array}$ \\
\hline Dieldrin & 1,2 & $\begin{array}{c}82^{f} \\
(77-87)\end{array}$ & $\begin{array}{c}65^{\mathrm{e}} \\
(62-68) \\
51^{\dagger} \\
(47-55)\end{array}$ & $\begin{array}{c}75 \\
(74-76)\end{array}$ & $\begin{array}{c}109 \\
(78-121)\end{array}$ \\
\hline Endrin & 1,2 & $\begin{array}{c}83^{f} \\
(77-92)\end{array}$ & $\begin{array}{c}63^{\mathrm{e}} \\
(61-64) \\
39^{\mathrm{f}} \\
(33-45)\end{array}$ & $\begin{array}{c}89 \\
(88-89)\end{array}$ & $\begin{array}{c}107 \\
(75-127)\end{array}$ \\
\hline
\end{tabular}

a Sistema $A: 20 \mathrm{~mL}$ de hexano; $\mathrm{n}=4$ análises.

b Sistema B: $20 \mathrm{~mL}$ de hexano:diclorometano $(9,5: 0,5 \mathrm{v} / \mathrm{v}) ; \mathrm{n}=2$ análises.

c Sistema C: $10 \mathrm{~mL}$ de hexano:diclorometano $(9: 1 \mathrm{v} / \mathrm{v}) ; \mathrm{n}=2$ análises.

${ }^{d}$ Sistema D: $10 \mathrm{~mL}$ de hexano:diclorometano (8:2 v/v); $\mathrm{n}=4$ análises.

e 1’ fração: $10 \mathrm{~mL}$.

f $2^{\mathrm{a}}$ fração: $10 \mathrm{~mL}$.

Considerando a complexidade da matriz estudada e que, recentemente, alguns autores $(8,15)$ demonstraram erros quantitativos advindos do efeito matriz, dados preliminares de recuperação foram obtidos com soluções padrão preparadas em hexano e em extratos da matriz. A Tabela 2 mostra que os valores médios de recuperação, alcançados com soluções preparadas em hexano (91 a 105\%), foram superiores àqueles obtidos com extrato da amostra de própolis sólida (84 a 86\%) para todos os pesticidas estudados. Entretanto, os valores de desvio padrão relativo $(13,1$ a 16,8\%) não apresentaram diferenças significativas. Avaliando os dados obtidos para própolis (solução) verifica-se que os valores médios de recuperação (95 a 170\%) foram superiores, com soluções padrão 
preparadas em hexano para todos os pesticidas, principalmente, para Endrin (170\%). Os valores de desvio padrão relativo, obtidos com soluções preparadas em extrato da matriz (4,8 a 9,8\%), demonstraram maior reprodutibilidade em relação aos dados encontrados com soluções preparadas em hexano (7,2 a 27,8\%). Estes resultados estão de acordo com os dados obtidos por JIMÉNEZ et al. (8), os quais afirmaram que o efeito matriz depende não apenas da classe do pesticida (sendo o efeito mais pronunciado para organofosforados) como também do nível de fortificação estudado (quanto menor o nível, maior o efeito). Assim, com o objetivo de minimizar o efeito matriz, as amostras de própolis (sólida e solução) fortificadas nos níveis 0,6 e 1,2 $\mu \mathrm{g} / \mathrm{g}$ foram quantificadas com soluções padrão preparadas em extratos das amostras.

\section{TABELA 2 - COMPARAÇÃO DOS RESULTADOS DE RECUPERAÇÃO COM PADRÕES PREPARADOS EM SOLVENTE E EM EXTRATO DA MATRIZ}

\begin{tabular}{|c|c|c|c|c|c|}
\hline Pesticida & $\begin{array}{c}\mathrm{N} \text { vel de } \\
\text { Fortifica }{ }^{\mathrm{a}} \mathrm{O} \\
(\mu \mathrm{g} / \mathrm{g})\end{array}$ & Recu & $\begin{array}{l}\operatorname{pera}^{a} 0^{a} \\
(\%)\end{array}$ & $\begin{array}{l}\text { MØdia }^{a} \\
(\%)\end{array}$ & $\begin{array}{c}\text { Desvio Padra o Relativo } \\
(\%)\end{array}$ \\
\hline \multirow[t]{2}{*}{ Heptacloro } & $0,6^{b}$ & $\begin{array}{c}103 \\
91\end{array}$ & $128^{\mathrm{d}}$ & $\begin{array}{l}116 \\
100\end{array}$ & $\begin{array}{c}10,0 \\
7,6\end{array}$ \\
\hline & $1,2^{\circ}$ & $\begin{array}{l}73 \\
74\end{array}$ & $\begin{array}{l}110^{d} \\
101^{e}\end{array}$ & $\begin{array}{l}94 \\
86\end{array}$ & $\begin{array}{l}16,6 \\
15,1\end{array}$ \\
\hline \multirow[t]{2}{*}{ Aldrin } & $0,6^{b}$ & $\begin{array}{l}85 \\
86\end{array}$ & $\begin{array}{l}103^{d} \\
100^{e}\end{array}$ & $\begin{array}{l}95 \\
93\end{array}$ & $\begin{array}{l}7,2 \\
7,3\end{array}$ \\
\hline & $1,2^{\mathrm{c}}$ & $\begin{array}{l}69 \\
71\end{array}$ & $108^{d}$ & $\begin{array}{l}91 \\
85\end{array}$ & $\begin{array}{l}16,8 \\
15,9\end{array}$ \\
\hline \multirow[t]{2}{*}{ Dieldrin } & $0,6^{b}$ & $\begin{array}{l}87 \\
88\end{array}$ & $\begin{array}{l}109^{d} \\
97^{e}\end{array}$ & $\begin{array}{l}98 \\
92\end{array}$ & $\begin{array}{l}9,7 \\
4,8\end{array}$ \\
\hline & $1,2^{\circ}$ & $\begin{array}{l}81 \\
71\end{array}$ & $\begin{array}{l}116^{d} \\
101^{e}\end{array}$ & $\begin{array}{c}100 \\
84\end{array}$ & $\begin{array}{l}15,6 \\
16,0\end{array}$ \\
\hline \multirow[t]{2}{*}{ Endrin } & $0,6^{b}$ & $\begin{array}{c}123 \\
82\end{array}$ & $\begin{array}{l}221^{d} \\
102^{e}\end{array}$ & $\begin{array}{c}170 \\
92\end{array}$ & $\begin{array}{c}27,8 \\
9,8\end{array}$ \\
\hline & $1,2^{\circ}$ & $\begin{array}{l}84 \\
70\end{array}$ & $\begin{array}{l}119^{d} \\
102^{e}\end{array}$ & $\begin{array}{l}105 \\
85\end{array}$ & $\begin{array}{l}13,1 \\
16,6\end{array}$ \\
\hline
\end{tabular}

\footnotetext{
${ }^{a} \mathrm{n}=5$ análises.

${ }^{b}$ própolis (solução).

c própolis (sólida).

d comparação com solução padrão preparada em hexano.

e comparação com solução padrão preparada em extrato da matriz.
} 
Os resultados das análises de recuperação estão apresentados nas Tabelas 3 e 4. Valores médios de recuperação entre 79 e $100 \%$ foram obtidos para todos os pesticidas estudados. Os níveis de fortificação foram definidos por comparação com dados da literatura (8). A reprodutibilidade e a sensibilidade do método desenvolvido foram confirmadas pelos valores de desvio padrão relativo (2,7 a 16,6\%) e pelos valores de limite de detecção do método $(0,1 \mathrm{a} 0,4 \mu \mathrm{g} / \mathrm{g})$, calculados conforme CABRAS et al. (16). As análises cromatográficas foram realizadas sob as condições descritas em, aproximadamente, 32 min.

\section{TABELA 3 - EFICIÊNCIA DA RECUPERAÇÃO EM MATRIZ DE PRÓPOLIS SÓLIDA}

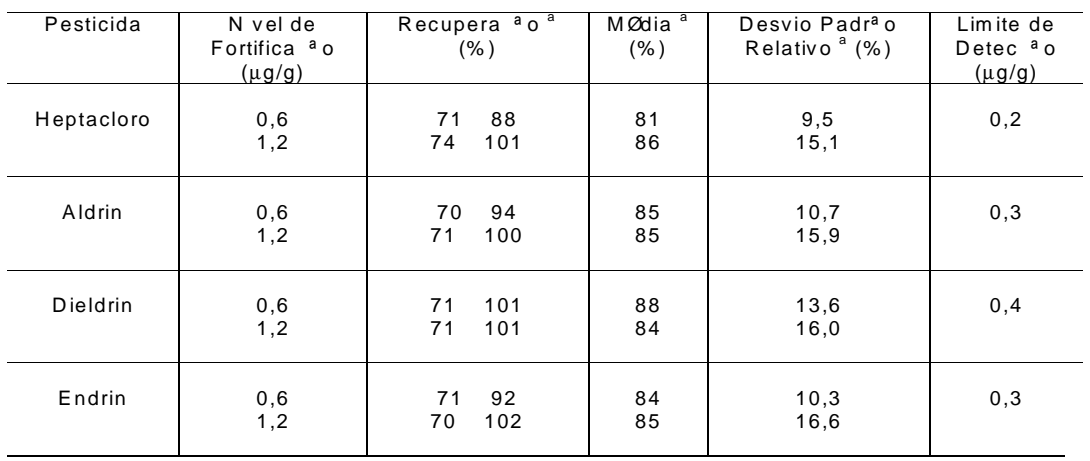

${ }^{a} \mathrm{n}=5$ análises.

\section{TABELA 4 - EFICIÊNCIA DA RECUPERAÇÃO EM MATRIZ DE PRÓPOLIS SOLUÇÃO}

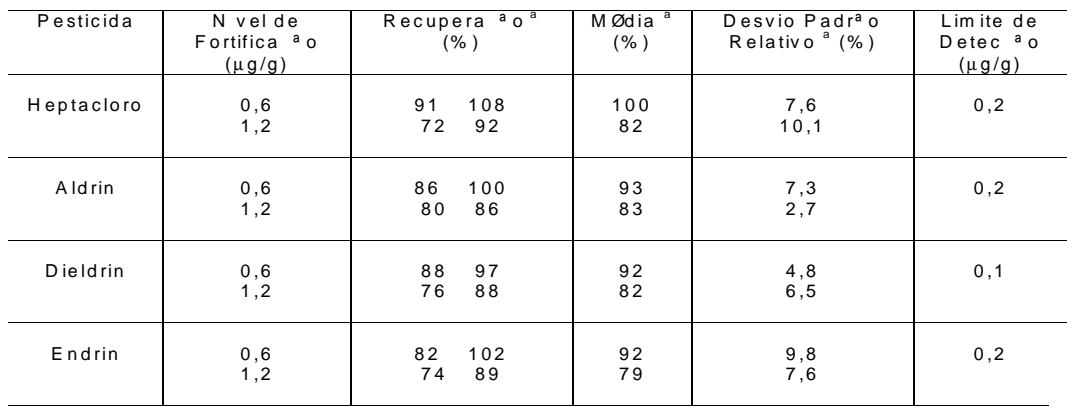

${ }^{a} \mathrm{n}=5$ análises. 


\title{
4 CONCLUSÃo
}

O procedimento analítico desenvolvido para determinação de Heptacloro, Aldrin, Dieldrin e Endrin em própolis (sólida e solução) mostrouse adequado para quantificação dos pesticidas estudados, tendo os resultados das recuperações evidenciado significativo efeito matriz. Resultados apropriados de recuperação (exatidão), situados entre 79 e $100 \%$ e de desvio padrão relativo (precisão) entre 2,7 e 16,6 \% foram obtidos, demonstrando a eficiência do método, cujos valores de limite de detecção situaram-se entre 0,1 e $0,4 \mu \mathrm{g} / \mathrm{g}$.

\begin{abstract}
METHOD FOR THE DETERMINATION OF HEPTACHLOR, ALDRIN, DIELDRIN AND ENDRIN IN PROPOLIS

A method for the determination of Heptachlor, Aldrin, Dieldrin and Endrin in propolis (solid and solution) was developed. After the samples homogenization with Celite the pesticides extraction was carried out with hexane and the extract was purified in Florisil cartridge. The pesticides elution was processed with hexane:dichloromethane $(9: 1 \mathrm{v} / \mathrm{v})$ and the concentrated eluate was analysed using gas-liquid chromatography with electron capture detection (GC-ECD). The efficiency of the proposed method was demonstrated by mean recoveries, ranging from 79 to $100 \%$ with relative standard deviation values between 2.7 to $16.6 \%$, obtained in the recovery analysis with fortified samples at levels of 0.6 and $1.2 \mu \mathrm{g} / \mathrm{g}$
\end{abstract}

KEY-WORDS: HEPTACHLOR; ALDRIN; DIELDRIN; ENDRIN; PROPOLIS.

\section{REFERÊNCIAS}

1 PRÓPOLIS. Disponível em: <http://sites.uol.com.br/torreecilha/ propolis/propolis. html>. Acesso em: 31 jan. 2000.

2 BREVES noções sobre os produtos das abelhas. Disponível em: <http://www.geocities.com/Eureka/Park/4579/sobremel.html>. Acesso em: 31 jan. 2000.

3 PEREIRA, P.C.M.; BARRAVIERA, B.; BURINI, R.C.; SOARES, A.M.V.C.; BERTANI, M.A. Use of honey as nutritional and therapeutic supplement in the treatment of infectious diseases. Disponível em: <http://www.cevap. org.br/revistas/jvat295/report.htm>. Acesso em: 31 jan. 2000. 
4 A REALEIRA. Própolis. Disponível: <http://www.arealeira.com.br/ propolis.htm>. Acesso em: 31 jan. 2000.

5 BERNAL, J. L.; del NOZAL, M. J.; TORIBIO, L.; JIMÉNEZ, J. J.; ATIENZA, J. High-performance liquid chromatographic determination of benomyl and carbendazim residues in apiarian samples. Journal of Chromatography A, v. 787, n.1/2, p. 129-136, 1997.

6 MUIINO, M.A.F.; LOZANO, J.S. Simplified method for the determination of organochlorine pesticides in honey. Analyst, v.116, n. 3, p. 269$271,1991$.

7 CABRAS, P.; MELIS, M.; SPANEDDA, L. Determination of cymiazole residues in honey by liquid chromatography. Journal of AOAC International, v. 76, n. 1, p. 92-94, 1993.

8 JIMÉNEZ, J. J.; BERNAL, J. L.; del NOZAL, M. J.; TORIBIO, L.; MARTÍN, M. T. Gas chromatography with electron-capture and nitrogen-phosphorus detection in the analysis of pesticides in honey after elution from a Florisil column - Influence of the honey matrix on the quantitative results. Journal of Chromatography A, v. 823, n. 1/2, p. 381-387, 1998.

9 DREYFUSS, M. F.; LOTFI, H.; MARQUET, P.; DEBORD, J.; DAGUET, J. L.; LACHÂTRE, G. Analyse de résidues de pesticides dans des miels et des pommes par CLHP et CPG. Analysis, v. 22, n. 5, p. 273-280, 1994.

10 SPITTLER, T. D.; MARAFIOTI, R. A.; HELFMAN, G. W.; MORSE, R. A. Determination of Carbaryl in honeybees and pollen by highperformance liquid chromatography. Journal of Chromatography, v. 352, p. 439-443, 1986.

11 THIER, H. P.; ZEUMER $H$. Manual of pesticide residue analysis. Weinheim, Germany: VCH, 1987. v. 1, p. 37-44.

12 POLESE, L.; MINELLI, E. V.; JARDIM, E. F. G.; RIBEIRO, M. L. Small-scale method for the determination of selected organochlorine pesticides in soil. Fresenius J. Anal. Chem., v. 354, n. 4, p. 474476, 1996.

13 KATO, M. H.; NAVICKIENE, S.; POLESE, L.; JARDIM, E. F. G.; 
RIBEIRO, M. L.; MINELLI, E. V. Metodologia rápida e eficiente para análise de pesticidas organoclorados em fubá. Química Nova, v. 19, n. 6, p. 620-622, 1996.

14 HAMAKAMI, M. M.; POLESE, L.; JARDIM, E. F. G.; RIBEIRO, M. L. Extração e "clean up"em uma única etapa para determinação de resíduos de pesticidas organoclorados em lanolina. Pesticidas: Revista de Ecotoxicologia e Meio Ambiente, v. 7, p. 69-76, jan./ dez.1997.

15 BERNAL, J. L.; del NOZAL, M. J.; JIMÉNEZ, J. J.; RIVERA, J. M. Matrix effects in the determination of acaricides and fungicides in must by gas chromatography with electron-capture and nitrogenphosphdgorus detection. Journal of Chromatography A, v. 778, $n$. 1/2, p. 111-117, 1997.

16 CABRAS, P.; ANGIONI, A.; GARAU, V. L.; MELIS, M.; PIRISI, F. M.; CABITZA, F.; DEDOLA, F.; NAVICKIENE, S. Determination of buprofezin, pyridaben, and tebufenpyrad residues by gas chromatography-mass-selective detection in clementine citrus. J. Agric. Food Chem., v.46, p.4255-4259, 1998. 